\title{
Aerial Vehicles Tracking Using Noncoherent Crowdsourced Wireless Networks
}

\author{
Hazem Sallouha ${ }^{1}$, Alessandro Chiumento ${ }^{2}$, and Sofie Pollin ${ }^{1}$ \\ ${ }^{1}$ Department of Electrical Engineering, KU Leuven, Belgium \\ ${ }^{2}$ EEMCS Faculty, University of Twente, The Netherlands \\ Email: hazem.sallouha@esat.kuleuven.be
}

\begin{abstract}
Air traffic management (ATM) of manned and unmanned aerial vehicles (AVs) relies critically on ubiquitous location tracking. While technologies exist for AVs to broadcast their location periodically and for airports to track and detect AVs, methods to verify the broadcast locations and complement the ATM coverage are urgently needed, addressing anti-spoofing and safe coexistence concerns. In this work, we propose an ATM solution by exploiting noncoherent crowdsourced wireless networks (CWNs) and correcting the inherent clock-synchronization problems present in such non-coordinated sensor networks. While CWNs can provide a great number of measurements for ubiquitous ATM, these are normally obtained from unsynchronized sensors. This article first presents an analysis of the effects of lack of clock synchronization in ATM with CWN and provides solutions based on the presence of few trustworthy sensors in a large non-coordinated network. Secondly, autoregressivebased and long short-term memory (LSTM)-based approaches are investigated to achieve the time synchronization needed for localization of the AVs. Finally, a combination of a multilateration (MLAT) method and a Kalman filter is employed to provide an anti-spoofing tracking solution for AVs. We demonstrate the performance advantages of our framework through a dataset collected by a real-world CWN. Our results show that the proposed framework achieves localization accuracy comparable to that acquired using only GPS-synchronized sensors and outperforms the localization accuracy obtained based on state-of-the-art CWN synchronization methods.
\end{abstract}

Index Terms-Localization, tracking, multilateration, synchronization, Kalman filter, dynamic clock model, TDoA, unmanned aerial vehicle (UAV)

\section{INTRODUCTION}

Global air traffic of manned and unmanned aerial vehicles (AVs) is on a steady rising trend, projecting unmanned aerial vehicles (UAVs) to outgrow manned aerial vehicles (MAVs) by several orders of magnitude over the next 20 years [1]. With UAVs entering the civil airspace, air traffic management (ATM) must expand to handle their coexistence with MAVs, ensuring safe airspace with ubiquitous tracking capabilities [2]-[7]. Unlike the MAVs safety system, which includes onboard navigation aids, a pilot to intervene, and ground ATM, UAVs safety systems rely primarily on ground ATM due to size and power limitations [7]. In fact, UAV ATM becomes particularly crucial in beyond visual line of sight and autonomous missions.

The essence of ground ATM is AVs' location information typically acquired through radio frequency (RF)-based surveil-

Hazem Sallouha is funded by PDM Internal Funds - KU Leuven lance systems. The location information can be obtained independently from the AV using primary surveillance radar (PSR), and in an AV-dependent manner using automatic dependent surveillance-broadcast (ADS-B) with AV's global positioning system (GPS)-based location. While existing surveillance systems are sufficient for MAVs' ATM, they are anticipated to face fundamental challenges when dealing with airspace featuring both MAVs and UAVs [4]-[6]. First, radar-based solutions have a fixed coverage radius, and the small UAV cross-section poses great detection limitations [56]. Secondly, location-broadcast-based methods, such as ADS-B, are expected to suffer from serious reliability and security risks with UAVs scaling up the number of AVs in the airspace [2 4]. Besides, broadcast methods are broadly vulnerable to spoofing attacks [8 [9]. Alternatively, crowdsourced ATM is gaining considerable research focus, as it addresses coverage limitations and promises better resilience against malicious attacks [6]-[9].

Crowdsourced ATM exploits the large-scale deployments of crowdsourced wireless networks (CWNs) to capture AVs' broadcast messages, enabling localization using time differences of arrival (TDoA)-based multilateration (MLAT) [7|10]. TDoA-based MLAT, as an independent means of localization, does not require any information from the target AV, which facilitates the detection of spoofing attacks. Currently deployed CWNs rely on off-the-shelf software-defined radio (SDR)enabled receivers to sense the spectrum and forward the collected data to the network backend via the Internet. While CWNs' setup guarantees a smooth expansion of the network coverage, it results in a network with noncoherent receivers, consisting of a mix of synchronized and unsynchronized ones [11]. However, localization using TDoA-based MLAT requires all receivers involved in the localization process to be synchronized [12].

The tunable SDR used in CWN receivers enables attractive synchronization opportunities by relying on reference signals from existing wireless networks, e.g., Wi-Fi beacons, LTE synchronization messages, or ADS-B messages from trusted aircraft. In this article, we tackle the synchronization problem in noncoherent CWNs by exploiting the ADS-B messages from registered commercial aircraft, along with the limited subset of synchronized receivers, to realize the synchronization needed for TDoA-based MLAT localization. This promising potential of a crowdsourced-based AV localization and tracking, alongside the insisting demand on secure and ubiquitous 
ATM, inspired our work in this article.

\section{A. Related Works}

Recently, the localization and tracking problem of AVs in general, and UAVs in particular, has been studied in several articles. The approaches proposed in these articles are broadly categorized into radar systems and localization systems based on RF signal emitted from the target [13]. In [5], Zheng et al. investigated the detection and localization problem of UAV swarms using a radar method based on the Dechirpkeystone transform and frequency-selective reweighted trace minimization. Guerra et al. [14] introduced an aerial monostatic dynamic radar network distributed on multiple UAVs to localize and track any malicious UAV in the surrounding area. While both [5] and [14] provided a promising accuracy, it is practically infeasible to deploy such systems for country-wide UAV tracking. On the one hand, it is economically challenging considering the high-cost of radar systems [6], and on the other hand, it might be inappropriate or even forbidden to deploy a radar system in urban areas due to the relatively high-power used.

Localization methods based on broadcast RF signals from $\mathrm{AVs}$ offer a cost-efficient alternative with the ability to provide country-wide coverage by exploiting the large-scale deployments of CWNs [7 11 15]. Predominantly, these localization methods employ MLAT based on the received signal strength (RSS) [16], time of arrival (ToA) [17], or TDoA [12]. In [6], Yang et al. introduced a cost-efficient crowdsourcing system to detect and localize UAVs. It has been shown that by exploiting the Wi-Fi beacons broadcast from UAVs, it is possible to detect and subsequently localize them using RSS. However, the considered range was limited to $400 \mathrm{~m}$; scaling up this range will significantly reduce the localization accuracy, which is inversely proportional to the true distance between the UAV and the ground anchor [13[18]. Furthermore, RSS-based and ToA-based methods require knowledge about the transmit power and the transmit time used by the target $\mathrm{AV}$, which are not available in the case of a spoofing target [13]. Such knowledge is not necessary for TDoA-based methods, making it a favorable choice for MLAT with CWN [10[19]. In [19], Seo et $a l$. proposed a particle filter-based 3D target tracking algorithm with measurement fusion of TDoA, frequency difference of arrival (FDoA), and angle of arrival (AoA). Strohmeier et al. [7] introduced a grid-based localization approach for AVs using the $k$-nearest-neighbor $(k-\mathrm{NN})$ algorithm with TDoA measurements at a CWN. The reported results in both [7] and [19] assumed CWNs with time-synchronized receivers. However, since CWNs use noncoherent receivers [1120], this assumption excludes the majority of $\mathrm{CWN}$ receivers that are not time-synchronized, resulting in sparse CWN coverage. For instance, in the OpenSky network, the percentage of timesynchronized receiver sensors is only $15 \%$ of the total number of sensors [15[20].

The noncoherent and widely-distributed nature of CWNs restrains them from establishing applications such as cooperative signal decoding and cooperative localization [21]. An autoregressive (AR)-based approach, joint with a Kalman filter
(KF), has been adopted in [22|23] to model the clock behavior in devices with low-precision clocks. While the AR process enables promising clock models, the provided results were based on co-located sensors in a single indoor or outdoor environment. In addition, a reference broadcast signal for synchronization was available on-demand. Calvo-Palomino et al. [24] proved that CWNs could achieve a certain level of synchronization, sufficient for cooperative signal decoding, by using synchronization messages from existing LTE infrastructure. However, this method requires resynchronization with every received message. This requirement is overwhelming for the CWN backend, and for the receivers that have limited processing power.

Another promising synchronization reference for noncoherent CWNs is the ADS-B messages from registered commercial aircraft with their trusted broadcast positions. The work presented in [25] exploits these trusted ADS-B messages in conjunction with the limited subset of synchronized receivers to model and subsequently compensate for the clock offset based on an AR model. Such clock offset compensation suggests a great potential to achieve the synchronization needed to enable accurate TDoA-based MLAT. Nevertheless, the results presented in [25] were only preliminary, lacking details on the clock offset behavior, AR model analysis, and the overall localization performance. Moreover, the framework structure and its workflow were not presented.

\section{B. Contribution and Article Structure}

This article addresses AVs' localization problem using widely distributed noncoherent CWNs, aiming at large-scale and anti-spoofing crowdsourced-based ATM. We consider a real-life CWN with receivers classified into 15\% of GPSenabled sensors (GSNs) with GPS-synchronized clocks and $85 \%$ of non-GPS sensors (SNs) with drifting clocks that are not synchronized. We investigate the clock offset of the SN receivers, allowing them to engage in the TDoA-based MLAT process. We also extend and refine our previous work [25] by introducing a full localization system design and detailing the workflow for processing both training and test data. Furthermore, we introduce a novel machine-learning-based clock offset modeling, along with comprehensive analyses, concerning the performance of both clock offset modeling and AV localization. The main contributions and merits of this article are summarized as follows:

- We propose a novel localization framework and a full localization system design, enabling $\mathrm{AV}$ s tracking using a noncoherent CWN with a mix of SN and GSN receivers. The proposed system presents a large-scale RF-based ATM solution, which can be used to verify the broadcast location of both manned and unmanned vehicles, enabling spoofing attacks detection and tracking.

- The proposed localization framework investigates two synchronization approaches for noncoherent CWNs: ARbased and long short-term memory (LSTM)-based. We rely on broadcast messages from trusted AVs and the few available GSN to characterize the dynamic el of the SNs. Subsequently, the resulting model is used to compensate 
for SNs' clock offset, enabling target AVs tracking with TDoA-based MLAT, joint with a KF.

- Finally, a measurement-based dataset collected from a real-world CWN is used to assess the performance of the proposed localization framework. Our results confirm the effectiveness of the proposed framework, minimizing the localization error by around $50 \%$ compared to instantaneous SNs synchronization using their prior measured offsets.

The rest of the article is organized as follows. In Section II. we present the system model and the TDoA method. Subsequently, the clock offset model is detailed in Section III. Section IV presents our proposed localization framework with noncoherent CWNs. Subsequently, we present our experimental results in Section V] Finally, Section VI concludes this article.

Notation: Italic letters, simple bold letters, and capital bold letters represent scalars, vectors, and matrices, respectively. $\left(a_{1}, a_{2}, \ldots\right)$ represents a sequence and $\left[a_{1}, a_{2}, \ldots\right]^{\mathrm{T}}$ represents a column vector, with $[.]^{\mathrm{T}}$ being the transpose operator. We use $\tilde{x}, x^{-}$, and $\hat{x}$ to denote the estimate (or the prediction), the a priori estimate, and the KF-based estimate, of $x$, respectively.

\section{SYSTEM MODEL}

This section presents the noncoherent CWN model considered in this work. Subsequently, it introduces the TDoA-based MLAT method employed in the proposed framework.

\section{A. Crowdsourced Network Model}

Consider a ground-based crowdsourced ATM network deployed to localize and track AVs, as illustrated in Fig. 11 We assume that AVs send periodic RF signals which are received by $N$ receivers, denoted by $R x_{1}, R x_{2}, \ldots, R x_{N}$ where the $i$ th receiver $R x_{i} \in\{\mathrm{GSN}, \mathrm{SN}\}$ with $i=1,2, \ldots, N$. The receivers register the signal's ToAs, and subsequently, forward them to a centralized station where the location of the corresponding AV is estimated. To develop a suitable localization method, it is crucial first to identify the characteristics of the crowdsourced ATM networks. In particular, the communication channel and the receivers' characteristics.

1) Communication Channel Characteristics: The communication channel between AVs and ground terminals depends on the propagation environment and the location of the AV with respect to the ground terminal. A widely adopted airto-ground channel model [26 27] is presented in [28]. As reported in this work, the line-of-sight $(\mathrm{LoS})$ and non-lineof-sight (NLoS) links are considered separately, along with their probabilities of occurrence, and are expressed as

$$
\mathrm{PL}_{n}=20 \log \left(\frac{4 \pi f d}{c}\right)+\mu_{n}, \quad n \in[\operatorname{LoS}, \mathrm{NLoS}],
$$

where $f$ is the carrier frequency, $c$ is the speed of light, $\mu_{\text {LoS }}$ denotes the mean excessive path loss, and $d$ is the direct distance between the AV and the ground terminal, given by $d=\sqrt{h^{2}+r^{2}}$. The probability of having a LoS link

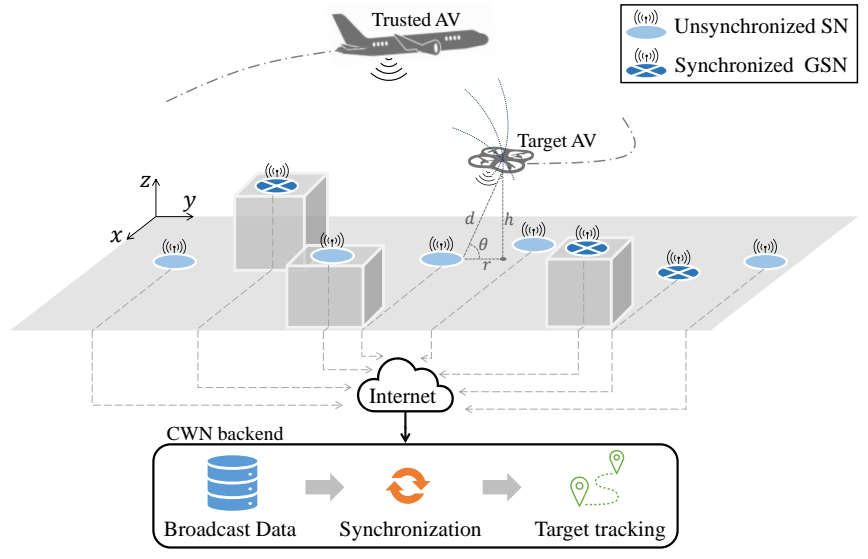

Fig. 1: Target AVs tracking using a noncoherent CWN with broadcast messages from trusted AVs used as a synchronization reference.

fundamentally depends on the environment, including density and height of buildings, as well as the elevation angle between the AV and the ground terminal. Accordingly, the expression of the LoS probability can be written as [26 28]

$$
\mathcal{P}_{\mathrm{LoS}}=\frac{1}{1+a_{o} \exp \left(-b_{o} \theta\right)},
$$

where $a_{o}$ and $b_{o}$ are environment dependent constants [28] and $\theta$ is the elevation angle shown in Fig. 11. The NLoS probability is simply $\mathcal{P}_{\mathrm{NLoS}}=1-\mathcal{P}_{\mathrm{LoS}}$. Intuitively, for cases where $h>>r$, and $r \neq 0$, we have $\theta \rightarrow 90^{\circ}$, resulting in a $\mathcal{P}_{\text {LoS }}$ that converges to one with roughly free-space path loss model [13]. For instance, commercial aircraft experience such high LoS probability as they fly well-above buildings. While low-altitude AVs might encounter NLoS links, they generally experience a LoS probability significantly higher than that of ground-to-ground scenario [13 26 28$]$.

In terms of range, the channel can be classified as a long-range communication channel. In the case of a rotarywing $\mathrm{AVs}$, the range varies from a few hundreds of meters to kilometers. This range typically increases by orders of magnitude in the case of fixed-wing AVs.

2) Receivers Characteristics: Crowdsourced ATM networks aim at providing global coverage that is not limited to airports and airfields. To this end, a massive number of receivers with various off-the-shelf hardware are used, e.g., Radarcape, SBS-3, and GRX1090 [15]. From the TDoA localization perspective, receivers can be categorized as synchronized, namely GSN, and unsynchronized, namely SN. The GSN receivers are usually GPS synchronized, meaning that they are constantly resynchronized to compensate for any clock offset. These receivers use a GPS disciplined oscillator (GPSDO) as a stable time reference for their local clocks [29]. Moreover, GSN receivers' timestamps have a rather high resolution. Unsynchronized $\mathrm{SN}$ receivers, on the other hand, are subject to (sometimes heavy) drifting, and their timestamps have a lower resolution compared to GSNs. For instance, in the OpenSky network [15], GSNs and SNs have resolutions of about $40-60 \mathrm{MHz}$ and $12 \mathrm{MHz}$, respectively. 


\section{B. MLAT Location Estimation Method}

The MLAT process establishes a set of equations relating the TDoA measurements, the receivers' positions, and the unknown target position. The approaches used for solving the MLAT' set of equations can be classified as statistical, numerical, and algebraic [10]. The statistical approaches are highly dependent on the environment's statistical characteristics and are considered open-form algorithms, as they do not provide a closed-form solution [30]. Numerical approaches can introduce a closed-form solution; however, a formally driven parameter from the target is required for the numerical approximation [10]. Consequently, in the following, we adopt an algebraic approach that uses neither statistical assumptions nor numerical approximations [12], offering an elegant closedform solution.

Consider a CWN employing MLAT localization based on TDoA measurements among its distributed receivers. The TDoA associated with the $i$ th receiver, $R x_{i}$ and the $j$ th receiver, $R x_{j}$, is $t_{j}-t_{i}$, where $t_{i}$ and $t_{j}$ are the ToAs at $R x_{i}$ and $R x_{j}$ respectively. Accordingly, one can define the difference in distance $d_{i j}$ as

$$
\begin{aligned}
d_{i j} & :=d_{i}-d_{j} \\
& =\left(t_{i}-t_{o}\right) c-\left(t_{j}-t_{o}\right) c=\left(t_{i}-t_{j}\right) c,
\end{aligned}
$$

where $c$ is the speed of light, $t_{o}$ is the AV clock time, and $d_{i}$ and $d_{j}$ are the distances from the AV to $R x_{i}$ and $R x_{j}$, respectively. The distance from the AV to the $i$ th receiver is expressed as

$$
d_{i}=\sqrt{\left(x_{i}-x\right)^{2}+\left(y_{i}-y\right)^{2}+\left(z_{i}-z\right)^{2}},
$$

where $(x, y, z)$ is the $\mathrm{AV}$ position and $\left(x_{i}, y_{i}, z_{i}\right)$ is the position of $R x_{i}$ with $i=1,2, \ldots, N$. Without loss of generality, the origin of the Cartesian coordinate system is set at $R x_{1}$, i.e., $\left(x_{1}, y_{1}, z_{1}\right)=(0,0,0)$. Consequently, using (4), $d_{1}$ can be written as

$$
d_{1}=\sqrt{x^{2}+y^{2}+z^{2}} .
$$

Now, given that $d_{i 1}=d_{i}-d_{1}$, 4 can be rewritten in terms of $d_{i 1}$ as

$$
\left(d_{i 1}+d_{1}\right)^{2}=D_{i}^{2}-2 x_{i} x-2 y_{i} y-2 z_{i} z+d_{1}^{2},
$$

where

$$
D_{i}^{2}=x_{i}^{2}+y_{i}^{2}+z_{i}^{2} .
$$

Expanding (6), one obtains

$$
-x_{i} x-y_{i} y-z_{i} z=d_{i 1} d_{1}+\frac{1}{2}\left(d_{i 1}^{2}-D_{i}^{2}\right) \text {. }
$$

In order to localization a target in $3 \mathrm{D}$, we need $N \geq 4[10]$. Let us define the target position as $\mathbf{q}:=[x, y, z]^{\mathrm{T}}$, following (8) we can express the problem in matrix format as

$$
\mathbf{B q}=d_{1} \mathbf{m}+\mathbf{c},
$$

where $\mathbf{B}, \mathbf{m}$, and $\mathbf{c}$ are respectively expressed as

$$
\mathbf{B}=\left[\begin{array}{ccc}
x_{2} & y_{2} & z_{2} \\
x_{3} & y_{3} & z_{3} \\
\vdots & \vdots & \vdots \\
x_{N} & y_{N} & z_{N}
\end{array}\right], \mathbf{m}=\left[\begin{array}{c}
-d_{21} \\
-d_{31} \\
\vdots \\
-d_{N 1}
\end{array}\right], \mathbf{c}=\frac{1}{2}\left[\begin{array}{c}
D_{2}^{2}-d_{21}^{2} \\
D_{3}^{2}-d_{31}^{2} \\
\vdots \\
D_{N}^{2}-d_{N 1}^{2}
\end{array}\right]
$$

Following [12 31], the position of the AV, defined as $\tilde{\mathbf{q}}:=$ $[\tilde{x}, \tilde{y}, \tilde{z}]^{\mathrm{T}}$, can be obtained by solving (9) for $\mathbf{q}$, which gives

$$
\tilde{\mathbf{q}}=\left(\mathbf{B}^{\mathrm{T}} \mathbf{B}\right)^{-1} \mathbf{B}^{\mathrm{T}}\left(d_{1} \mathbf{m}+\mathbf{c}\right) \text {. }
$$

Equation 10 contains $d_{1}$, which is unknown. Substituting (10) into (5) yields a quadratic equation in $d_{1}$. Solving for $d_{1}$ and substituting the positive root back into $(10)$ gives the final solution for $\tilde{\mathbf{q}}$. In the case of two positive solutions for $d_{1}$, we choose the one that lies in the domain of interest, e.g., the one that presents a positive altitude [31]. To obtain an accurate position, all receivers need to be synchronized. However, in crowdsourced ATM networks, receivers are of two categories: GSNs, with clocks that are constantly GPS synchronized, and SNs, with clocks that are subject to drifts. In the next section we present the clock modeling and highlight its main design parameters.

\section{Clock Modeling AND Noise Analysis}

The broadcast signal from the AV is received by multiple receivers, among which some are unsynchronized. Therefore, it is crucial to model the clock's behavior to compensate for any offset. The time difference with the actual time, at the $k$ th sample index, is called time offset $\eta_{[k]}$, and the instantaneous clock offset rate of change is known as clock skew $\lambda_{[k]}$. In fact, the instantaneous time offset depends on the instantaneous clock skew $\lambda_{[k]}$, and the previous clock offset. Accordingly, for a given receiver's clock, the discrete-time clock offset model is expressed as

$$
\eta_{[k]}=\eta_{[k-1]}+\lambda_{[k-1]} \tau_{[k-1]}+\omega_{[k]},
$$

where $k$ is the sample index, $\tau_{k}$ is the $k$ th sampling period 1 and $\omega_{[k]}$ is a zero-mean normally distributed noise. Following (11), we identify two influential parameters in the clock model, the clock skew $\lambda_{[k-1]}$ and the additive noise $\omega_{[k]}$.

\section{A. Clock Skew}

Clock skew results in a clock that runs at a varying speed compared to the actual time. This varying speed is attributed to the oscillator noise, which varies with the supply voltage, age, temperature, and other environmental factors, resulting in a time-varying skew with certain randomness. In general, the oscillator noise is characterized as a nonstationary process; however, the influential parameters' range of change, such as temperature and humidity, varies relatively slowly with time, resulting in a quasi-stationary process [23 32]. Therefore, it is of utmost importance to keep track of the quasi-stationarity behavior when modeling the clock skew. In fact, the timeseries stationarity characteristics define how often we need to re-calibrate the skew model.

Time-series-based models are used to represent the timevarying skew over an hours-long timescale [23]. Such recursive models must be designed to capture the dynamic as well as the stochastic clock behavior. Once the skew, $\lambda_{[k]}$, is estimated, we exploit the model in (11) to predict the clock offset, and subsequently, compensate for it in TDoA calculations.

\footnotetext{
${ }^{1}$ The sampling period $\tau_{[k-1]}$ is the difference in time between the $k$ th and the $(k-1)$ th samples
} 


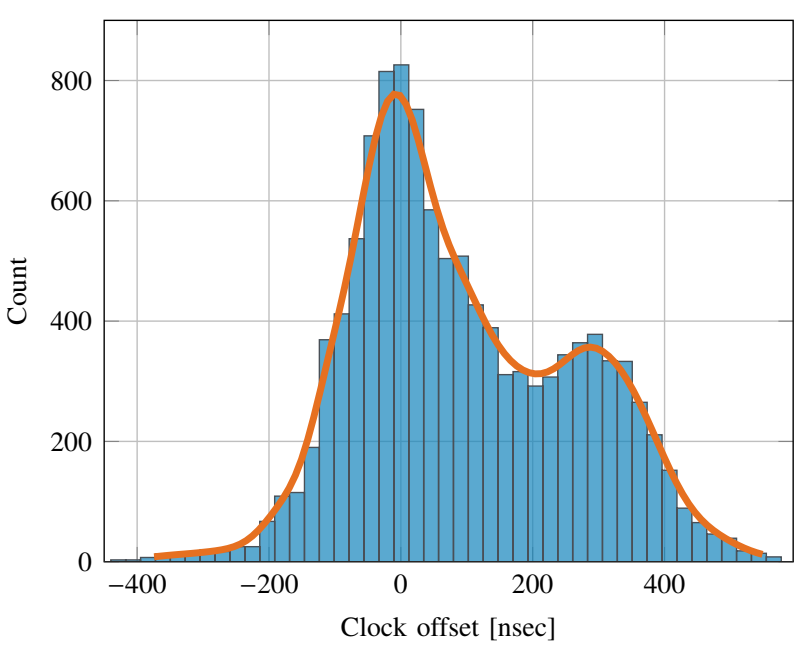

Fig. 2: The clock offset histogram of an $\mathrm{SN}$ receiver measured with respect to a GSN receiver by relying on common ADS-B messages from trusted AVs, as given in 12.

\section{B. Clock Noise and Offset Error}

In addition to the clock skew, the additive offset noise has a noticeable effect on the clock modeling precision. In fact, the vast majority of clock models in the literature assume a normally distributed clock noise [22[23]. Adding the normally distributed noise to the time-varying clock skew, results in a clock offset with a clear tenancy towards a multimodal distribution. In fact, clocks often drift differently depending on the influential parameter [33]. For instance, the instantaneous clock skew could vary at different rates with the receiver's printed circuit board temperature, which changes with the processing load. Thus, the same clock could have different clock drift rates on different occasions, resulting in a clock offset with a multimodal distribution.

Figure 2 illustrates the histogram of the clock offset in an $\mathrm{SN}$ receiver with respect to a GSN one. The figure depicts a bimodal error distribution, indicating a skew process with multiple influential parameters [23]. Such non-Gaussian distribution restrains the use of conventional TDoA solutions and Bayes filtering algorithms, which are typically designed assuming a Gaussian-type error [34]. Therefore, after compensating for the offset, it is essential to track the residual clock error distribution to make sure that the non-Gaussian distribution is eliminated and that it follows a normal distribution.

\section{AV TRACKING USING CWNS}

In this section, we introduce our localization and tracking framework using noncoherent CWNs. Figure 3 depicts the block diagram of the proposed framework. As shown in the figure, it consists of three main blocks: the data preparation block, the synchronization block, and the localization block. In the following, we detail each block's role in the AVs tracking process.

\section{A. Data Preparation Block}

The broadcast messages in noncoherent CWNs, arriving from various AVs, are captured by two types of sensors:
GSN and SN. This block is mainly responsible for preprocessing and sorting the collected data as well as feeding it to the relevant subsequent block. First, the captured messages' sources must be distinguished as either trusted AVs or target AVs. Subsequently, the received messages are sorted based on the receiver's type, namely GSN or SN. Messages arriving from trusted AVs are used in constructing the training data as they carry AV's location information, speed, and identification. In particular, the proposed framework utilizes trusted AVs' broadcast messages mutually received by GSNs and SNs to construct the training data for SN clock offset modeling. To this end, consider a trusted AV's broadcast message received by a GSN receiver and an SN receiver. Given the locations of both receivers and the trusted AV location, we can express the measured clock offset as

$$
\eta_{[k]}=t_{\mathrm{GSN}[k]}-\Delta_{\mathrm{GSN}[k]}+\Delta_{\mathrm{SN}[k]}-t_{\mathrm{SN}[k]},
$$

where $t_{\mathrm{GSN}[k]}$ and $t_{\mathrm{SN}[k]}$ are the measured ToAs at the GSN receiver and $\mathrm{SN}$ receiver, respectively, $\Delta_{\mathrm{GSN}[k]}$ and $\Delta_{\mathrm{SN}[k]}$ denote the broadcast message's time of flight (ToF) from the $\mathrm{AV}$ to the GSN and SN receivers, respectively. Note that the ToF can be easily measured by dividing the distance between the trusted $\mathrm{AV}$ and the receiver, as given in (4), over the speed of light, $c$. Following (12), we construct the training dataset, which is fed to the synchronization block where the clock offset model of the corresponding SN receiver is estimated. Now, messages from target AVs can be exploited to estimate their locations or verify the broadcast ones. Messages received from the target AV by GSNs are passed directly to the MLAT process in the localization block, whereas those received by SNs must first pass through the synchronization block, as illustrated in Figure 3

\section{B. Synchronization Block}

This block models the clock of SNs and compensates for any clock offset. It takes the ToA registered at a given SN, from the target AV broadcast, as an input. Besides, it also exploits the ToAs recorded at both SNs and GSNs, from trusted AVs, as training references. Since commercial AVs send ADS-B messages every second, one might intuitively suggest using the prior measured offset to compensate for the subsequent clock offset. Despite the wide availability of ADS-B messages, less than $35 \%$ of these messages are received by more than one receiver due to the low reliability of ADS-B messages in urban areas [2]. In addition, receiving the ADS-B message by multiple receivers does not necessarily mean having a GSN one to act as a reference in measuring the clock offset of other SNs. As a result, consecutive clock offset measurements are separated by relatively long time gaps, e.g., several minutes. Modeling the clock offset enables predicting the clock offset during these time gaps, which guarantees the continuity of the localization service. The proposed synchronization block investigates two approaches to model the clock offset and synchronize SNs' ToA measurements: AR and LSTM-based recurrent neural network (RNN).

1) Autoregressive-Based Synchronization: When using the recursive model in (11) to model the clock offset, one needs 


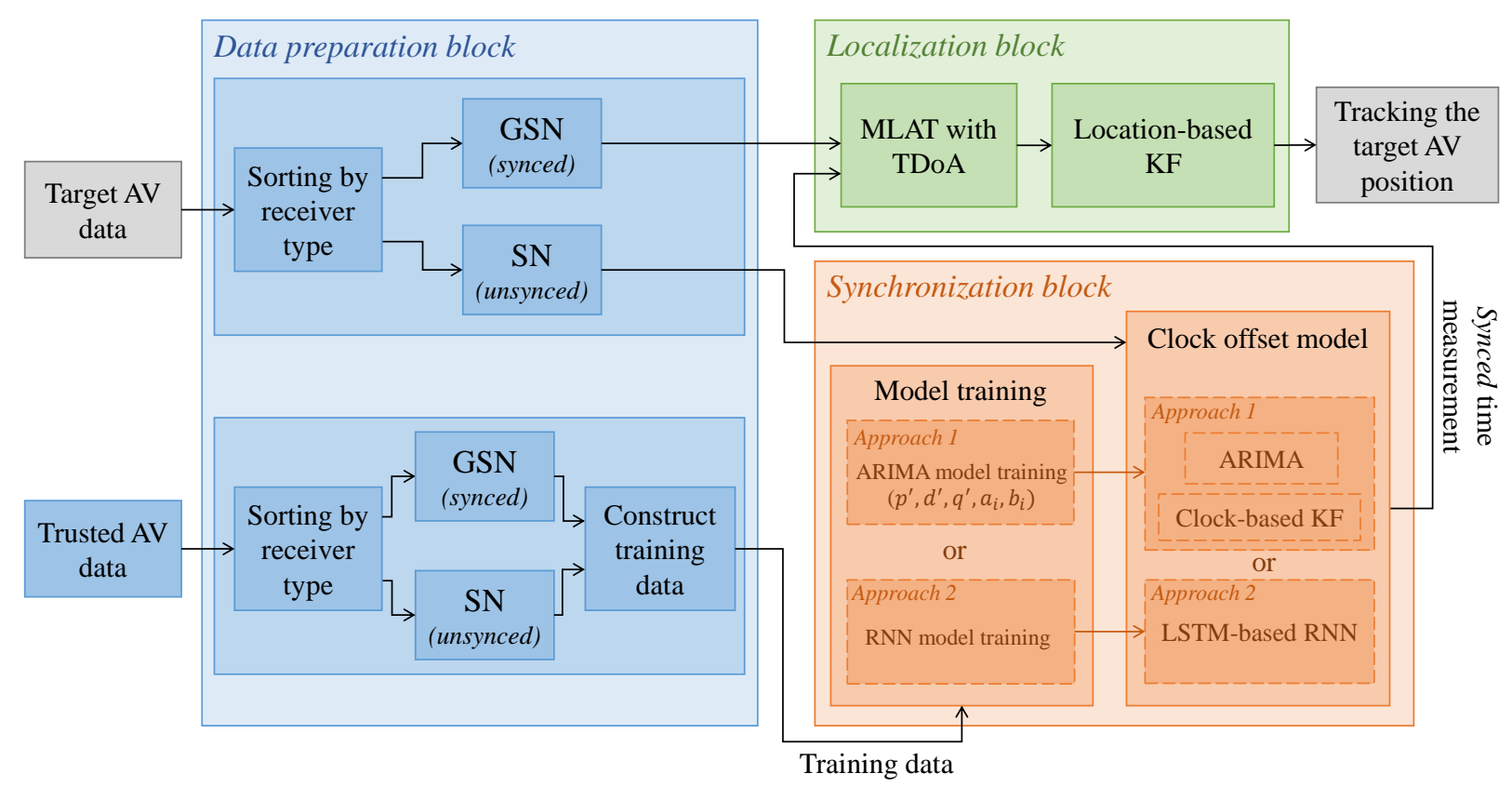

Fig. 3: The block diagram of the proposed AV localization framework illustrating the two clock synchronization approaches considered. Broadcast messages from trusted AVs are exploited to train clock offset models, enabling both GSNs and SNs to engage in the target AV localization.

to measure the skew, $\lambda_{[k]}$. The clock skew is time-varying; however, for a given measurement duration, it can be modeled using an AR process [23]. In particular, autoregressive integrated moving average (ARIMA) considers the dynamic as well as the stochastic behavior of the clock, and is confirmed suitable for modeling clock skew [22[23]. Consider an ARIMA model, with orders $p^{\prime}, d^{\prime}$, and $q^{\prime}$; the skew can be represented by

$$
\underbrace{\left(1-\sum_{i=1}^{p^{\prime}} a_{i} L^{i}\right)}_{\text {autoregressive }} \underbrace{(1-L)^{d^{\prime}}}_{\text {integrated }} \lambda_{[k]}=\underbrace{\left(1+\sum_{i=1}^{q^{\prime}} b_{i} L^{i}\right) \xi_{[k]}}_{\text {moving average }},
$$

where $L$ is the lag operator ${ }^{2}, \xi_{[k]}$ denotes a zero-mean Gaussian noise, $a_{i}$ 's and $b_{i}$ 's are the $\mathrm{AR}$ and the moving average coefficients, respectively. The model in (13) represents an $\operatorname{ARIMA}\left(p^{\prime}, d^{\prime}, q^{\prime}\right)$ process where $p^{\prime}, d^{\prime}$, and $q^{\prime}$ are nonnegative integers representing the order of the autoregressive model, the degree of differencing, and the order of the moving average, respectively. ARIMA models are particularly attractive for skew modeling because of their ability to handle any possible non-stationarity in the data [22 35]. The differencing degree $d^{\prime}$ is set in order to achieve stationarity in the timeseries dataset used for model training and testing. Applying the KPSS test for stationarity [36] reveals that, based on the receiver, a differencing degree of zero or one $d^{\prime}=[0,1]$ is sufficient to guarantee a stationary clock offset for all SN receivers of the CWN considered in this work. The orders of the autoregressive $p^{\prime}$ and the moving average $q^{\prime}$ can be identified using the Box-Jenkins methodology [37],

\footnotetext{
${ }^{2}$ The lag operator raised to the power $i$ implies that $L^{i} \lambda_{[k]}=\lambda_{[k-i]}$
}

TABLE I: RNN Model Architecture

\begin{tabular}{|c||l|l|}
\hline Layer index & \multicolumn{1}{|c|}{ Type } & \multicolumn{1}{c|}{ Details } \\
\hline \hline 1 & Sequence input & - \\
\hline \multirow{2}{*}{2} & \multirow{3}{*}{ LSTM } & Units: 10 \\
\cline { 3 - 3 } & & State activation: tanh \\
\cline { 3 - 3 } & & Gate activation: sigmoid \\
\hline 3 & Fully connected & Units: 5 \\
\hline 4 & Dropout & 0.2 \\
\hline 5 & Fully connected & Units: 1 \\
\hline 6 & Regression output & Mean squared error \\
\hline
\end{tabular}

which relies on the autocorrelation function (ACF) and partial ACF (PACF) of the time-series data ( $c f$. Subsection V-A). The identified state-space formulation of the ARIMA model, results from substituting (13) in (11), allows a KF formulation [22]. Therefore, as presented in [22] and [23], a KF is typically used in conjugation with the model presented in (11), to track the time-varying skew and offset, further mitigating any residual error of the ARIMA model.

2) LSTM-Based Synchronization: The LSTM network, a type of RNN, is known for its effective handling of long-term dependencies in time-series data [38]. The LSTM network has feedback connections, enabling predictions based on timedependent characteristics. Since the clock offset is by default a time-series process, LSTM network can learn its behavior over time, and subsequently, make predictions for future values.

The proposed RNN consists, first, from an LSTM layer with 30 hidden units for the time-series-based clock offset learning. Subsequently, the LSTM layer is followed a fully connected layer, a dropout layer and another fully connected layer. Finally, a regression output layer is presented, delivering the output of the clock offset learning model. The Adam optimizer [39], a first-order gradient-based optimizer, with a 
learning rate of 0.01 is used for clock model training. Table I details the architecture of the LSTM-based RNN model used in this work.

Clock offset models provide predictions for the clock behavior. In particular, we train the models so that we can predict $\eta_{[k]}$ based on $\eta_{[k-1]}$. However, the nondeterministic nature of the clock skew prevents a particular clock model from precisely tracking its behavior, resulting in a model's residual error. Consider the AR and the LSTM clock offset models, let $\tilde{\eta}_{[k]}$ denote the $k$-th sample model-based clock offset prediction; the residual error of the corresponding model is then expressed as

$$
e_{r[k]}=\eta_{[k]}-\tilde{\eta}_{[k]} \cdot
$$

where $\eta_{[k]}$ represents the true clock offset.

\section{Localization Block}

The localization block consists of the MLAT localization method, introduced in Subsection II-B followed by a KF. This combination of the MLAT and KF enables tracking of the target AV position over time. The movement of the AV is modeled as a dynamic system. Given the periodic transmissions from an $\mathrm{AV}$, the state vector at the $k$ th transmission is written as

$$
\mathbf{s}_{[k]}=\left[x_{[k]}, y_{[k]}, z_{[k]}, \dot{x}_{[k]}, \dot{y}_{[k]}, \dot{z}_{[k]}\right]^{\mathrm{T}} \in \mathbb{R}^{6},
$$

where the sequence $\left(x_{[k]}, y_{[k]}, z_{[k]}\right)$ denotes the position of the AV, and $\left(\dot{x}_{[k]}, \dot{y}_{[k]}, \dot{z}_{[k]}\right)$ represents its velocity. Moreover, the input of this dynamic system is represented by the AV's acceleration which, at the $k$ th transmission, is given by

$$
\mathbf{u}_{[k]}=\left[\ddot{x}_{[k]}, \ddot{y}_{[k]}, \ddot{z}_{[k]}\right]^{\mathrm{T}} \in \mathbb{R}^{3},
$$

where the sequence $\left(\ddot{x}_{[k]}, \ddot{y}_{[k]}, \ddot{z}_{[k]}\right)$ represents the acceleration of the AV. Now, assuming four receivers are available, one can use 10 to calculate the AV's position using TDoA. Consequently, we express the $k$ th measurement vector $\tilde{\mathbf{q}}_{[k]}$, which is the calculated position from (10), as

$$
\tilde{\mathbf{q}}_{[k]}:=\left[\tilde{x}_{[k]}, \tilde{y}_{[k]}, \tilde{z}_{[k]}\right]^{\mathrm{T}} \in \mathbb{R}^{3} .
$$

Finally, the dynamic system can be written as

$$
\begin{aligned}
\mathbf{s}_{[k]} & =\boldsymbol{\Phi}_{[k-1]} \mathbf{s}_{[k-1]}+\boldsymbol{\beta}_{[k-1]} \mathbf{u}_{[k-1]}+\mathbf{w}_{[k-1]}, \\
\tilde{\mathbf{q}}_{[k]} & =\mathbf{H} \mathbf{s}_{[k]}+\mathbf{v}_{[k]},
\end{aligned}
$$

where $\boldsymbol{\Phi}_{[k]} \in \mathbb{R}^{6 \times 6}$ is the state transition matrix, $\boldsymbol{\beta}_{[k]} \in \mathbb{R}^{6 \times 3}$ is the input matrix, $\mathbf{u}_{[k]} \in \mathbb{R}^{3}$ is the model input vector, and $\mathbf{H} \in \mathbb{R}^{3 \times 6}$ represents the measurement matrix. Moreover, in (18) and (19), vectors $\mathbf{w}_{[k]}$ and $\mathbf{v}_{[k]}$ represent the model and measurement noise, respectively. They are assumed to be independent and normally distributed, i.e.,

$$
\begin{aligned}
\mathbf{w}_{[k]} \sim \mathcal{N}\left(0, \mathbf{Q}_{[k]}\right) \text { with } \mathbf{Q}_{[k]}=\mathbb{E}\left[\mathbf{w}_{[k]} \mathbf{w}_{[k]}^{\mathrm{T}}\right], \\
\mathbf{v}_{[k]} \sim \mathcal{N}\left(0, \mathbf{R}_{[k]}\right) \text { with } \mathbf{R}_{[k]}=\mathbb{E}\left[\mathbf{v}_{[k]} \mathbf{v}_{[k]}^{\mathrm{T}}\right],
\end{aligned}
$$

where $\mathbb{E}[$.$] denotes the expected value. Now, the KF$ formulation including the predictions and the updates is expressed as [34]

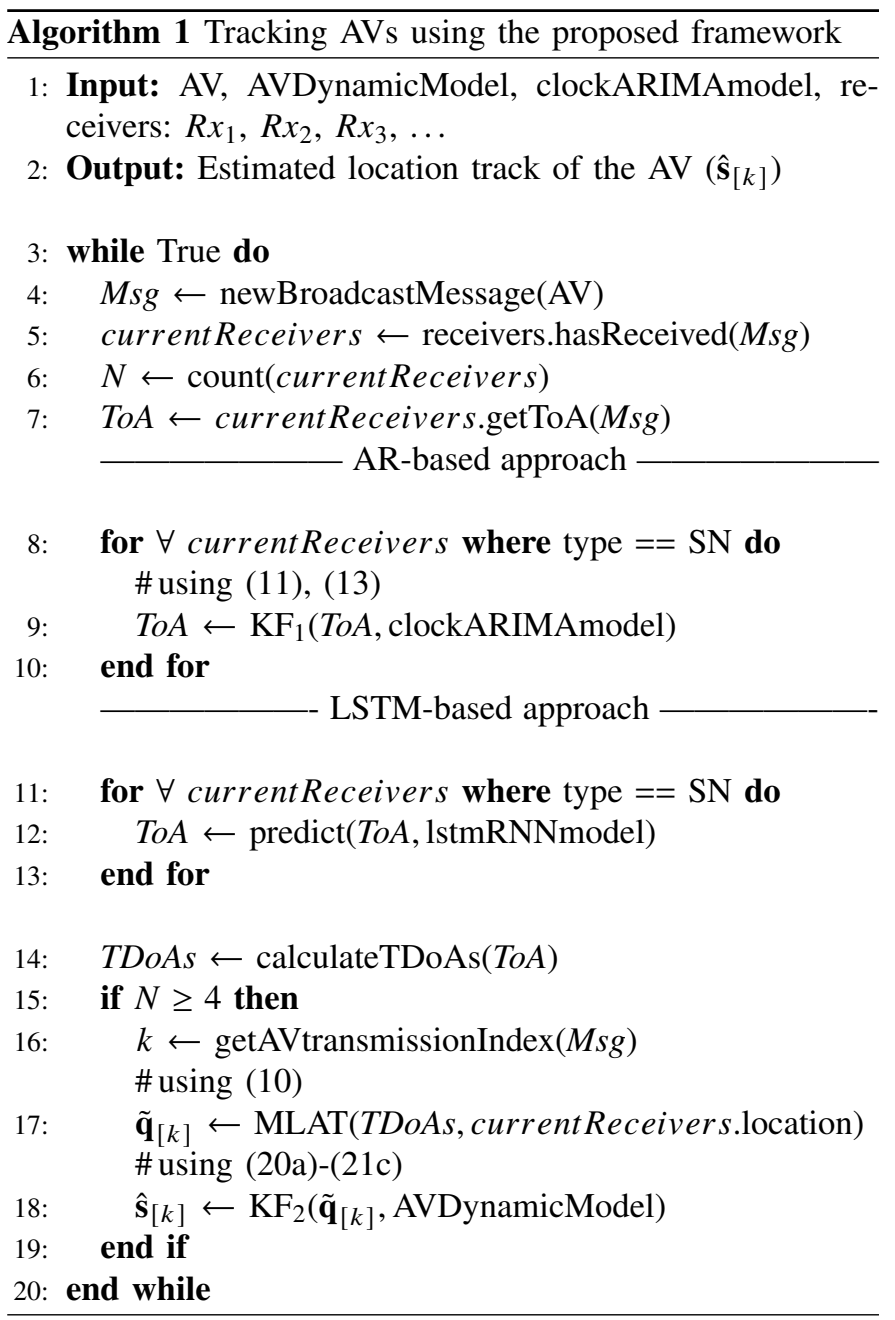

\section{Predict:}

$\hat{\mathbf{s}}_{[k]}^{-}=\boldsymbol{\Phi}_{[k-1]} \hat{\mathbf{s}}_{[k-1]}+\boldsymbol{\beta}_{[k-1]} \mathbf{u}_{[k-1]}$

$\mathbf{P}_{[k]}^{-}=\boldsymbol{\Phi}_{[k-1]} \mathbf{P}_{[k-1]} \boldsymbol{\Phi}_{[k-1]}+\mathbf{Q}_{[k-1]}$

Update:

$\mathbf{G}_{[k]}=\mathbf{P}_{[k]}^{-} \mathbf{H}^{\mathrm{T}}\left(\mathbf{H P}_{[k]}^{-} \mathbf{H}+\mathbf{R}_{[k]}\right)^{-1}$

$\hat{\mathbf{s}}_{[k]}=\hat{\mathbf{s}}_{[k]}^{-}+\mathbf{G}_{[k]}\left(\tilde{\mathbf{q}}_{[k]}-\mathbf{H} \hat{\mathbf{s}}_{[k]}^{-}\right)$

$\mathbf{P}_{[k]}=\left(\mathbf{I}-\mathbf{G}_{[k]} \mathbf{H}\right) \mathbf{P}_{[k]}^{-}$

where $\mathbf{I}$ is an identity matrix, $\mathbf{P}_{[k]} \in \mathbb{R}^{6 \times 6}$ is the state error covariance matrix, and $\mathbf{G}_{[k]} \in \mathbb{R}^{6 \times 3}$ is the KF gain. The KF presented in equations $20 \mathrm{a}-21 \mathrm{C}$ is able to overcome the Gaussian noise from the TDoA calculated position due to the clock offset noise.

In order to assess the performance of the proposed framework, we use the localization error as a performance metric. Considering a 3D Cartesian coordinate system, we defined the localization error as

$$
\mathcal{E}=\sqrt{(x-\hat{x})^{2}+(y-\hat{y})^{2}+((z-\hat{z}) / 10)^{2}},
$$

where $(x, y, z)$ is the true position of the $\mathrm{AV}$ and $(\hat{x}, \hat{y}, \hat{z})$ is the KF-based estimated one. In 22, we give less weight to 


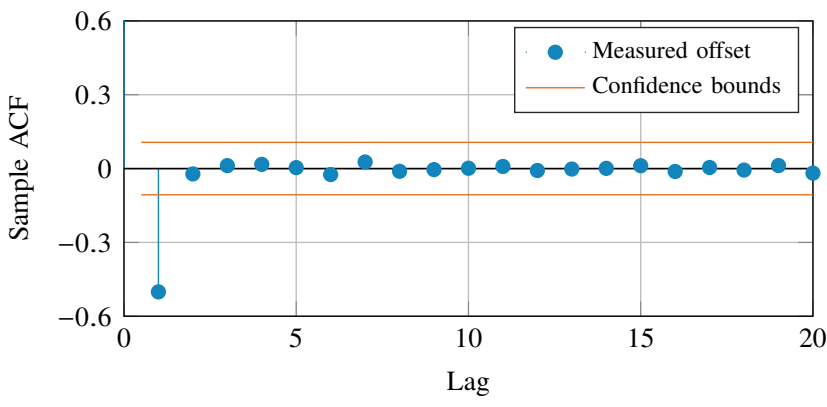

Fig. 4: The sample ACF of the measured clock offset with one degree of differencing $\left(d^{\prime}=1\right)$.

altitude estimation, since $x-y$ position is typically more critical than altitude estimation in tracking applications.

\section{Workflow and Algorithm Summary}

A summary of the proposed framework is presented in Algorithm 11. The algorithm works as follows. With each broadcast from AVs, the algorithm collects the ToA from all available receivers (currentReceivers). Subsequently, it checks the type of each receiver. For each unsynchronized receiver (i.e., type $==\mathrm{SN}$ ), it passes its data to the synchronization process, with the corresponding approach being either ARbased or LSTM-based. Once all ToAs are synchronized, they are used to calculate TDoAs, which are subsequently used to estimate the location $\left(\tilde{\mathbf{q}}_{[k]}\right)$ by employing MLAT. The estimated location is then processed using a $\mathrm{KF}$ (i.e., $\mathrm{KF}_{2}$ ) with the AV dynamic model to obtain the final location estimate $\left(\hat{\mathbf{s}}_{[k]}\right)$.

\section{EXPERIMENTAL RESULTS}

In this section, we assess the performance of the proposed method when applied on a dataset collected by the OpenSky Network [20]. The dataset consists of ADS-B messages sent from commercial aircraft and received by a total of 523 receivers, uniformly distributed around Europe, with 15\% of them are GSNs and the rest are SN receivers. The dataset is stored in different files, where each file represents a one-hour recording of ADS-B messages received by all 523 receivers. The stored data includes the actual time at the server, the aircraft ID, the number of available receivers for each message, and the receivers' information. The receiver information contains its ID, location, ToA, and type. Table II depicts a visualization of the dataset. In addition, the true locations of the aircraft are also available. The true position is used to calculate the localization error from (22). It is worth noting that although the considered dataset is based on MAV, the performance analysis is also valid for other AV. This due to the fact that the LoS probability $\mathcal{P}_{\text {LoS }}$ given in (2) approaches one at altitudes above the average buildings height [13], leading to a steady TDoA performance above this altitude. Moreover, similar to the ADS-B technology in MAVs, the majority of UAVs use Wi-Fi for communication [6], allowing CWNs to capture their Wi-Fi beacons [40].

To construct the training and the test datasets, we consider a one-hour recording of ADS-B messages mutually received

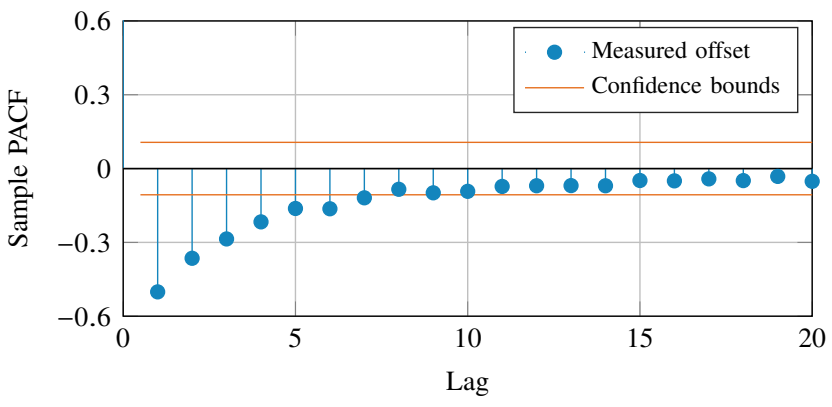

Fig. 5: The sample PACF of the measured clock offset with one degree of differencing $\left(d^{\prime}=1\right)$.

by four nodes, which corresponds to a total of approximately 12700 messages. First, we filtered out messages from eight aircraft and used them as a test dataset, representing the target AVs. The reaming dataset is assumed to be collected from trusted AVs, which are used to measure the clock offset of SNs using equation (12). Subsequently, we train both the LSTMbased RNN and the ARIMA models based on messages within a window of 20 minutes. The resulting models are then used to predict the clock offset for the subsequent time instants at which messages from the target AVs arrive. The models are retrained again after 20 minutes with an updated training data of the same size. This 20 minutes retraining period is chosen empirically to 1) guarantee a sufficient amount of common new messages (e.g., > 100 messages over a span of 20 minutes) between the SN and a GSN for effectual retraining, 2) bound the predictions' cumulative error, and 3) make sure that the clock offset measurements used in model training and testing represent a stationary process. The time required to train the clock offset models varies based on the size of the available training messages. For the considered ARIMA model, once $p^{\prime}, d^{\prime}$, and $q^{\prime}$ are identified, the training time ranges from 0.5 to 3 seconds, whereas for the LSTM-based model, the training time ranges from 30 to 60 second ${ }^{3}$ In the following, we first analyze the proposed clock synchronization approaches. Subsequently, the localization performance of the proposed framework is investigated.

\section{A. Synchronization Analysis}

To identify the order of the ARIMA model, we adopt the Box-Jenkins methodology [37], which recommends choosing the autoregressive order $p^{\prime}$ and the moving average order $q^{\prime}$ based on the number of lags that have values above the confidence bounds of the PACF and ACF, respectively. Figure 4 and Figure 5, respectively, present the ACF and PACF of an SN's clock offset with $90 \%$ concordance bounds. In both figures, we introduce one degree of differencing to make sure that the considered clock offset is a stationary process. The negative correlation in the figures is attributed to this differencing. Following Figure 4, we set $q^{\prime}=1$. Similarly, based on Figure 5, we infer an AR order $p^{\prime}=6$. Putting all together, the resulting model is $\operatorname{ARIMA}(6,1,1)$. Here, it is

\footnotetext{
${ }^{3}$ A desktop computer without a dedicated Graphics processing unit (GPU) is used for training the models considered in this work.
} 
TABLE II: Representation of the Dataset Structure

\begin{tabular}{cccccc}
\hline \hline & & \multicolumn{2}{c}{$R x_{1}$} & & $R x_{N}$ \\
\cline { 4 - 6 } Time [sec] & avID & $N$ & {$\left[\mathrm{ID}_{1}\right.$, location $\left._{1}, \mathrm{ToA}_{1}, \mathrm{type}_{1}\right]$} & $\ldots$ & {$\left[\mathrm{ID}_{N}\right.$, location $\left._{N}, \mathrm{ToA}_{N}, \mathrm{type}_{N}\right]$} \\
\hline $\mathrm{T}=0.5$ & 630 & 5 & {$[12,(46.6810,7.6653,10), 121 . ., \mathrm{GSN}]$} & $\ldots$ & {$[22,(46.0810,7.2653,8), 123 . ., \mathrm{SN}]$} \\
$\mathrm{T}=1.2$ & 1033 & 4 & {$[63,(52.3564,4.9522,12), 113 . ., \mathrm{SN}]$} & $\ldots$ & {$[24,(44.0810,6.2653,20), 173 . ., \mathrm{SN}]$} \\
$\vdots$ & $\vdots$ & $\vdots$ & $\vdots$ & & $\vdots$ \\
\hline
\end{tabular}

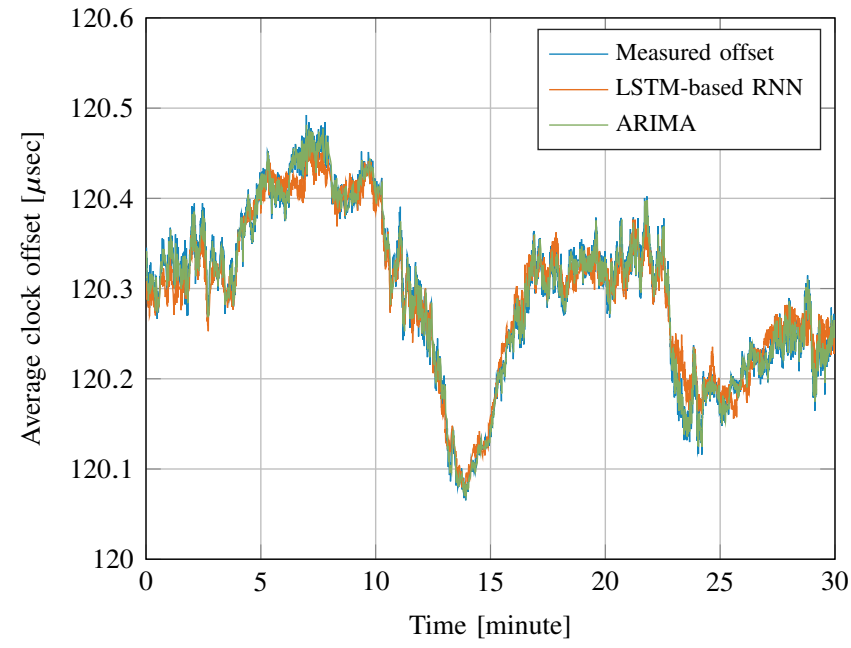

Fig. 6: A compression of clock modeling using LSTM-based RNN and ARIMA-based approaches. Both approaches achieve rather accurate modeling.

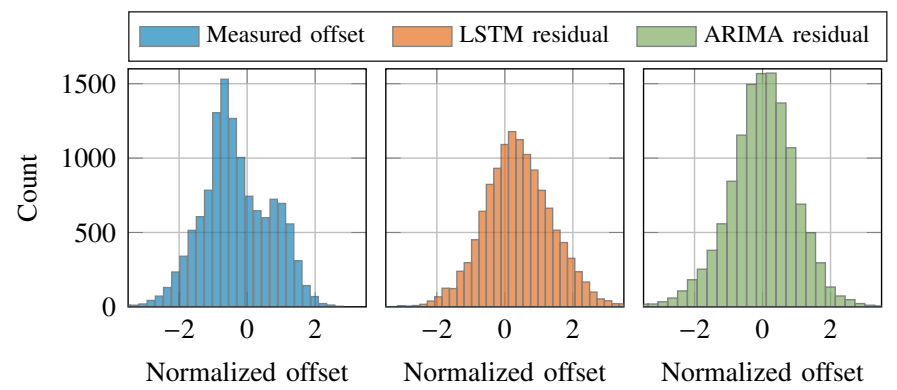

Fig. 7: Probability plot comparing the distribution of the clock offset residuals to the normal distribution.

worth noting that for the SNs considered in this work, where no differencing is needed to achieve stationarity, similar orders $q^{\prime}$ and $p^{\prime}$ are observed from the corresponding ACF and PACF plots, resulting in an $\operatorname{ARIMA}(6,0,1)$ model.

Figure 6 presents the clock offset modeling when using LSTM and ARIMA over 30 minutes period. As shown in the figure, both approaches provide a rather accurate offset modeling that ranges in the margin of 400 nanoseconds. As illustrated in the figure, both approaches confirmed their ability to cope with the clock offset, despite its nonlinearity and randomness.

As stated in Section III] it is crucial to make sure that the clock model's residual error follows a normal distribution. This normality is needed for the subsequent localization block. Figure 7 presents the histogram plots for an SN receiver's clock offset. The figure depicts the bimodal distribution of

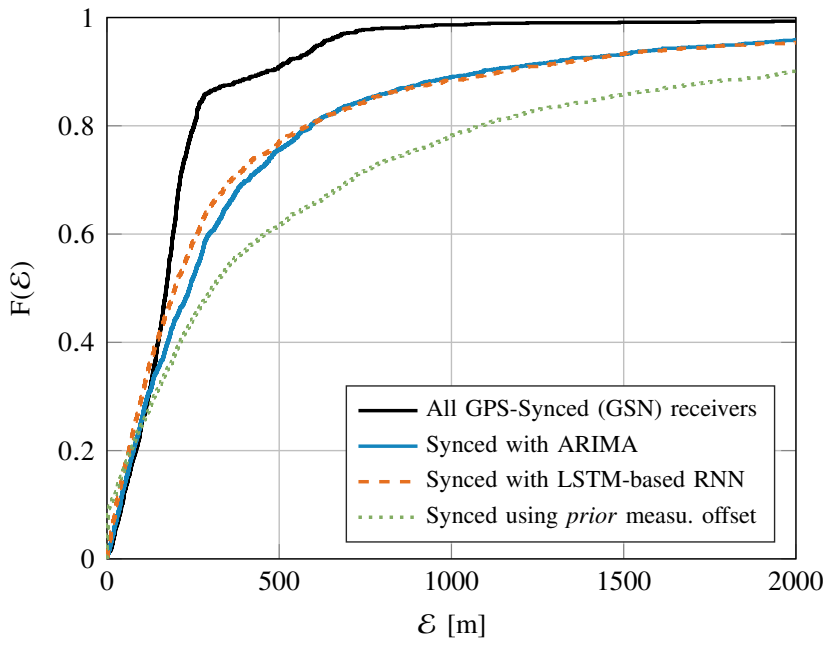

Fig. 8: The empirical CDF of the localization error with for both LSTM-based RNN and ARIMA-based synchronization approaches.

the normalized offset before any compensation. As shown in the figure, employing AR and LSTM models to predict and compensate for the clock offset results in residual errors $\left(e_{r[k]}\right.$ 's) with a bell-shaped normal distribution. Accordingly, it can be confirmed that both ARIMA and LSTM clock models are able to successfully eliminate the bimodal distribution, leading to normally distributed residual errors. Such normally distributed error can be handled by the KF in the subsequent localization block.

\section{B. Localization and Tracking}

Figure 8 demonstrate the empirical cumulative distribution function (CDF) of the localization error using the proposed framework. The figure represents the localization error based on 1250 received broadcasts, which correspond to eight aircraft broadcasting messages over 40 minutes period. In this scenario, we consider four receivers, two of which are unsynchronized, i.e., two GSNs and two SNs. Figure 8 compares between the two synchronization approaches considered. In addition, the figure presents a scenario in which all receivers are GPS-synchronized, i.e., GSN receivers. Analyzing the figure, several observations can be drawn.

1) First, the figure shows that the considered ARIMA and LSTM-based synchronization models roughly provide a matching performance in terms of the localization error probability. However, the figure exhibits a slight performance gain for the LSTM-based approach over the ARIMA-based approach. This gain is attributed to the 


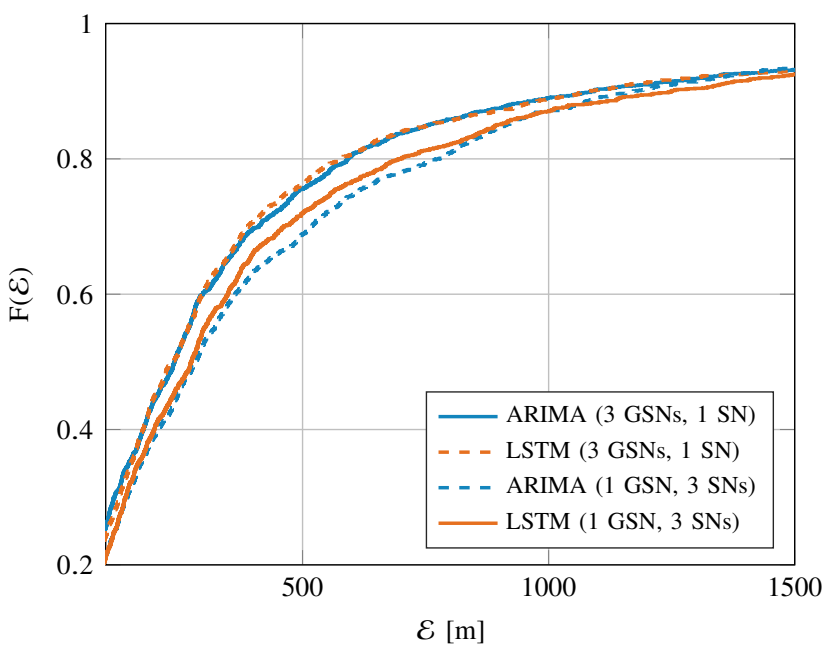

Fig. 9: The empirical CDF of the localization error with different numbers of GSNs and SNs.

structure of LSTM-based RNN that enables it to learn both long-term and short-term correlations and discard all the irrelevant ones in between [38].

2) Furthermore, Figure 8 proves the significant localization gain obtained by using the proposed framework. The proposed framework brings the localization performance nearly $50 \%$ closer to GSN receivers' performance compared to relying on the prior measured offset for synchronization without any clock modeling, similar to the synchronization method adopted in [24]. For instance, using the proposed framework's synchronization approaches, e.g., ARIMA or LSTM, brings the $80 \%$ error probability upper-bound from $1100 \mathrm{~m}$ using prior offset down to $600 \mathrm{~m}$.

3) Lastly, Figure 8 emphasizes that even with all receivers being GSP-synchronized, it is still possible to experience localization error in the range of hundreds of meters. This shows that the achieved accuracy has other limiting factors in addition to the clock synchronization. Examples of these factors are the receivers' orientation with respect to the target [7|12] and the packets' timetagging mechanize that may varies from one receiver to another [41].

Since the proposed framework relies on GSNs to synchronize SNs as well as to take part in the localization process, it is important to explore the impact of the proportion of GSNs in the CWN. The effect of the proportion of GSNs and SNs employed to localize the same target AVs from Figure 8 is presented in Figure 9 As the figure illustrates, the scenario with three GSNs and one SN outperforms the scenario where one GSN and three SNs are used. By comparing both scenarios, we confirm the positive impact of having more GSNs involved in the target localization. Nonetheless, the localization performance difference between the two scenarios is rather small, proving the considerable impact of the proposed synchronization methods. For instance, with LSTM-based synchronization, Figure 9 shows a roughly $3 \%$ difference in performance between the scenarios with one GSN

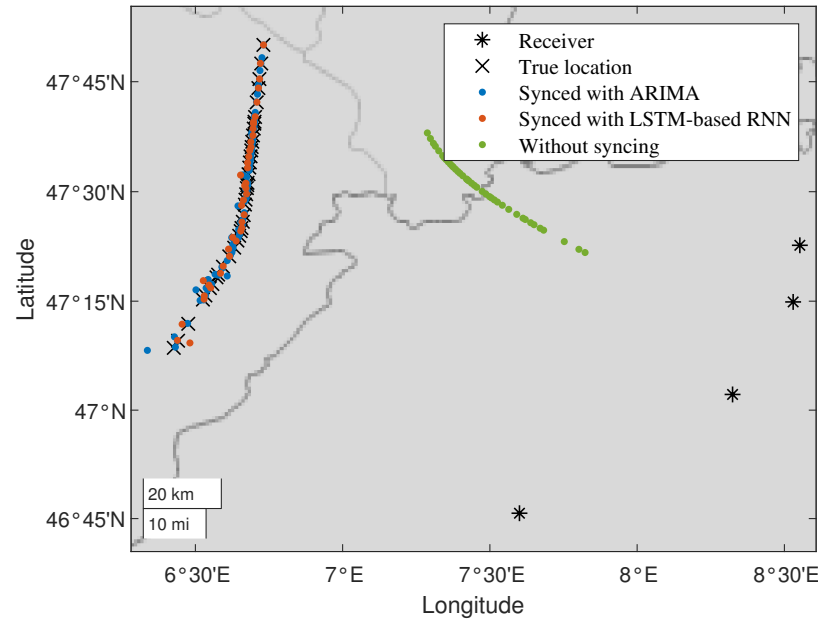

Fig. 10: The localization gains obtained by employing the proposed framework in a CWN with AV's trajectory parallel to the receivers' positions.

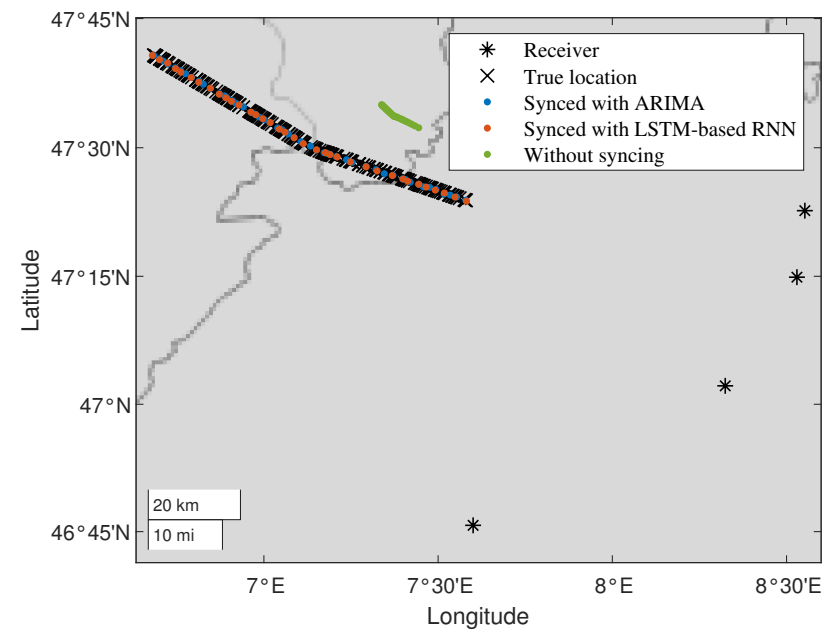

Fig. 11: The localization gains obtained by employing the proposed framework in a CWN with AV's trajectory perpendicular to the receivers' positions.

and three GSNs. In fact, this difference in performance with the proportion of employed GSNs and SNs is notably less pronounced compared to the effect of receivers' orientation with respect to the target AV (cf. Figure 10 and Figure 11).

The localization gains obtained with the proposed framework are also depicted in Figure 10 and Figure 11 . The figures exhibit both synchronization approaches, along with the case without synchronization. The MLAT cases without synchronization, presented in Figure 10 and Figure 11, illustrate the significant influence of the considered receivers' clock offset on deteriorating the localization performance before employing the proposed framework. Figure 10 presents an aircraft trajectory roughly parallel to the receiver's locations. The corresponding localization errors are summarized in Table III As shown in the table, using the ARIMA model to compensate for the clock offset decreases the error by orders of magnitude. Comparable performance is also achieved with LSTM-based synchronization. Furthermore, Table III highlights the effect of the location-based $\mathrm{KF}$ (i.e., $\mathrm{KF}_{2}$ ) on the localization performance, refining the performance of the TDoA-based MLAT process. 
TABLE III: Localization Error in Figure 10

\begin{tabular}{|l||c|c|}
\hline AV tracking method & Mean $\mathcal{E}[\mathrm{m}]$ & Median $\mathcal{E}[\mathrm{m}]$ \\
\hline \hline Without synchronization & $104 \times 10^{3}$ & $60 \times 10^{3}$ \\
\hline AR-based sync. without $\mathrm{KF}_{2}$ & 951 & 540 \\
\hline AR-based synchronization & 719 & 460 \\
\hline LSTM-based sync. without $\mathrm{KF}_{2}$ & 940 & 512 \\
\hline LSTM-based synchronization & 680 & 420 \\
\hline
\end{tabular}

TABLE IV: Localization Error in Figure 11

\begin{tabular}{|l||c|c|}
\hline AV tracking method & Mean $\mathcal{E}[\mathrm{m}]$ & Median $\mathcal{E}[\mathrm{m}]$ \\
\hline \hline Without synchronization & $30 \times 10^{3}$ & $24 \times 10^{3}$ \\
\hline AR-based sync. without $\mathrm{KF}_{2}$ & 200 & 108 \\
\hline AR-based synchronization & 160 & 105 \\
\hline LSTM-based sync. without $\mathrm{KF}_{2}$ & 210 & 106 \\
\hline LSTM-based synchronization & 155 & 103 \\
\hline
\end{tabular}

The performance of MLAT-based localization is considerably influenced by the target's position relative to the receivers' positions [7[12]. In Figure [11, we present another scenario, with the same set of receivers, where the AV's trajectory is perpendicular to the curve connecting the receivers' locations. As shown in Table IV] better performance is achieved with the same set of receivers and the same clock model. In particular, using the proposed framework with LSTM-based synchronization, an average localization error of $155 \mathrm{~m}$ is achieved. Similarly, comparable accuracy is obtained using the AR-based approach. In addition, it can be seen that even though the unsynchronized receives' performance improved by a factor of 3.4 in Table IV w.r.t. Table III synchronized ones improved by nearly a factor of 4.5 , showing the significance of the clock offsets corrected using the proposed framework. Furthermore, the mean localization errors of the proposed framework presented in Table III and Table IV shows a comparable performance to the $199 \mathrm{~m}$ mean error reported in [7]. However, unlike our proposed method which utilizes both GSN and SN receivers, the TDoA-based MLAT method in [7] only considers the GPS-synchronized GSN receivers.

The performance analysis of the proposed framework illustrates that LSTM-based and ARIMA-based methods provide comparable performances. In order to select one approach over the other, several aspects must be taken into account. The first aspect is the computational power since training LSTM models requires significantly more computational power than training ARIMA models. This article assumes that the model training is done at the network backend, which typically has sufficient computational power [11]. However, if a CWN opts for model training to run on the receiver's companion computer, then the ARIMA model is a more suitable approach. Another important aspect is the model tuning and updating over time as LSTM models provide a more sustainable and futureproof solution compared to ARIMA models. For all sensors considered in this work, we used the same LSTMbased RNN architecture, showing promising synchronization capabilities. On the contrary, choosing the ARIMA model's orders requires special attention to the stationarity, the amount of correlation, and the considered training period in the timeseries data, which all may vary from one receiver to another.

\section{CONCLUSION}

The localization of AVs using a partially unsynchronized CWN has been investigated. Particularly, a noncoherent CWN with a mix of synchronized and unsynchronized receivers has been considered. This paper proposed a framework for the localization and tracking of AVs, including spoofing ones. The proposed framework investigated two synchronization approaches for the noncoherent CWN: AR-based and LSTMbased. Subsequently, we used a TDoA-based MLAT, along with a KF, to estimate the location of the targeted AV. The proposed method has been validated using an OpenSky dataset, where it proved that both synchronization approaches were able to provide significant gains in terms of AVs localization accuracy, improving it by orders of magnitude. Finally, the reported results motivate investigating other deeplearning-based methods, such as convolutional neural networks (CNNs), with CWNs, where datasets recorded over several hours could potentially enable end-to-end location estimation.

\section{REFERENCES}

[1] US Department of Transportation, "Unmanned aircraft system (UAS) service demand 2015- 2035," Tech. Rep., 2013: https://fas.org/irp/program/collect/service.pdf, [online] Accessed on: June. 2020.

[2] S. Sciancalepore, S. Alhazbi, and R. Di Pietro, "Reliability of ads-b communications: Novel insights based on an experimental assessment," in Proceedings of the 34th ACM/SIGAPP Symposium on Applied Computing, pp. 2414-2421, 2019.

[3] O. Bekkouche, T. Taleb, and M. Bagaa, "Uavs traffic control based on multi-access edge computing," in 2018 IEEE Global Communications Conference (GLOBECOM), pp. 1-6, IEEE, 2018.

[4] A. Allouch, A. Koubâa, M. Khalgui, and T. Abbes, "Qualitative and quantitative risk analysis and safety assessment of unmanned aerial vehicles missions over the internet," IEEE Access, vol. 7, pp. 5339253410, 2019.

[5] J. Zheng, T. Yang, H. Liu, T. Su, and L. Wan, "Accurate detection and localization of uav swarms-enabled mec system," IEEE Transactions on Industrial Informatics, 2020.

[6] G. Yang, X. Shi, L. Feng, S. He, Z. Shi, and J. Chen, "Cedar: A costeffective crowdsensing system for detecting and localizing drones," IEEE Transactions on Mobile Computing, vol. 19, no. 9, pp. 2028-2043, 2020.

[7] M. Strohmeier, I. Martinovic, and V. Lenders, "A k-NN-based localization approach for crowdsourced air traffic communication networks," IEEE Transactions on Aerospace and Electronic Systems, vol. 54, no. 3, pp. 1519-1529, 2018.

[8] K. Jansen, M. Schäfer, D. Moser, V. Lenders, C. Pöpper, and J. Schmitt, "Crowd-GPS-Sec: Leveraging crowdsourcing to detect and localize GPS spoofing attacks," in 2018 IEEE Symposium on Security and Privacy (SP), pp. 1018-1031, IEEE, 2018.

[9] G. Liu, R. Zhang, C. Wang, and L. Liu, "Synchronization-free GPS spoofing detection with crowdsourced air traffic control data," in 2019 20th IEEE International Conference on Mobile Data Management (MDM), pp. 260-268, IEEE, 2019.

[10] I. A. Mantilla-Gaviria, M. Leonardi, G. Galati, and J. V. BalbastreTejedor, "Localization algorithms for multilateration (MLAT) systems in airport surface surveillance," Signal, Image and Video Processing, vol. 9, no. 7, pp. 1549-1558, 2015.

[11] S. Rajendran, R. Calvo-Palomino, M. Fuchs, B. Van den Bergh, H. Cordobés, D. Giustiniano, S. Pollin, and V. Lenders, "Electrosense: Open and big spectrum data," IEEE Communications Magazine, vol. 56, no. 1, pp. 210-217, 2017.

[12] A. H. Sayed, A. Tarighat, and N. Khajehnouri, "Network-based wireless location: challenges faced in developing techniques for accurate wireless location information," IEEE signal processing magazine, vol. 22, no. 4, pp. 24-40, 2005.

[13] M. M. Azari, H. Sallouha, A. Chiumento, S. Rajendran, E. Vinogradov, and S. Pollin, "Key technologies and system trade-offs for detection and localization of amateur drones," IEEE Communications Magazine, vol. 56, no. 1, pp. 51-57, 2018. 
[14] A. Guerra, D. Dardari, and P. M. Djurić, "Dynamic radar network of uavs: A joint navigation and tracking approach," IEEE Access, vol. 8, pp. 116454-116469, 2020.

[15] M. Schäfer, M. Strohmeier, V. Lenders, I. Martinovic, and M. Wilhelm, "Bringing up openSky: A large-scale ADS-B sensor network for research," in Proceedings of the 13th international symposium on Information processing in sensor networks, pp. 83-94, IEEE Press, 2014.

[16] H. Sallouha, A. Chiumento, S. Rajendran, and S. Pollin, "Localization in ultra narrow band IoT networks: design guidelines and tradeoffs," IEEE Internet of Things Journal, vol. 6, no. 6, pp. 9375-9385, 2019.

[17] Z. Abu-Shaban, X. Zhou, and T. D. Abhayapala, "A novel TOA-based mobile localization technique under mixed LOS/NLOS conditions for cellular networks," IEEE Transactions on Vehicular Technology, vol. 65 , no. 11 , pp. 8841-8853, 2016.

[18] H. Sallouha, M. M. Azari, and S. Pollin, "Energy-constrained uav trajectory design for ground node localization," in 2018 IEEE Global Communications Conference (GLOBECOM), pp. 1-7, IEEE, 2018.

[19] H. Seo, H. Kim, J. Kang, I. Jeong, W. Ahn, and S. Kim, "3D moving target tracking with measurement fusion of TDoA/FDoA/AoA," ICT Express, vol. 5, no. 2, pp. 115-119, 2019.

[20] OpenSky Network: https://opensky-network.org/, [online] Accessed on: Jun. 2020

[21] F. Harris, "Let's assume the system is synchronized," in Globalization of Mobile and Wireless Communications, pp. 311-325, Springer, 2011.

[22] W. Masood, J. F. Schmidt, G. Brandner, and C. Bettstetter, "DISTY: Dynamic stochastic time synchronization for wireless sensor networks," IEEE Transactions on Industrial Informatics, vol. 13, no. 3, pp. 14211429, 2017.

[23] H. Kim, X. Ma, and B. R. Hamilton, "Tracking low-precision clocks with time-varying drifts using Kalman filtering," IEEE/ACM Transactions on Networking (TON), vol. 20, no. 1, pp. 257-270, 2012.

[24] R. Calvo-Palomino, D. Giustiniano, V. Lenders, and A. Fakhreddine, "Crowdsourcing spectrum data decoding," in IEEE INFOCOM 2017 IEEE Conference on Computer Communications, pp. 1-9, IEEE, 2017.

[25] H. Sallouha, A. Chiumento, and S. Pollin, "G2A Localization: Aerial vehicles localization using a ground crowdsourced network," in Proceedings of the 7th OpenSky Workshop, vol. 67, pp. 37-43, 2019.

[26] M. Mozaffari, W. Saad, M. Bennis, Y.-H. Nam, and M. Debbah, "A tutorial on uavs for wireless networks: Applications, challenges, and open problems," IEEE communications surveys \& tutorials, vol. 21, no. 3, pp. 2334-2360, 2019.

[27] B. Alzahrani, O. S. Oubbati, A. Barnawi, M. Atiquzzaman, and D. Alg- hazzawi, "Uav assistance paradigm: State-of-the-art in applications and challenges," Journal of Network and Computer Applications, vol. 166, p. 102706, 2020.

[28] A. Al-Hourani, S. Kandeepan, and S. Lardner, "Optimal LAP altitude for maximum coverage," IEEE Wireless Communications Letters, vol. 3 , no. 6, pp. 569-572, 2014

[29] E. Kaplan and C. Hegarty, Understanding GPS: principles and applications. Artech house, 2005.

[30] I. A. Mantilla Gaviria, M. Leonardi, G. Galati, J. V. Balbastre Tejedor, and E. d. 1. Reyes Davó, "Efficient location strategy for airport surveillance using mode-s multilateration systems," International Journal of Microwave and Wireless Technologies, pp. 1-8, 2012.

[31] H. Schau and A. Robinson, "Passive source localization employing intersecting spherical surfaces from time-of-arrival differences," IEEE Transactions on Acoustics, Speech, and Signal Processing, vol. 35, no. 8 , pp. 1223-1225, 1987.

[32] K. J. Son and T. G. Chang, "Distributed nodes-based collaborative sustaining of precision clock synchronization upon master clock failure in ieee 1588 system," Sensors, vol. 20, no. 20, p. 5784, 2020.

[33] J. H. Funck, Synchronous data acquisition with wireless sensor networks, vol. 4. Universitätsverlag der TU Berlin, 2018.

[34] G. Welch, G. Bishop, et al., "An introduction to the Kalman filter," 1995.

[35] R. J. Hyndman and G. Athanasopoulos, Forecasting: principles and practice. OTexts, 2018

[36] D. Kwiatkowski, P. C. Phillips, P. Schmidt, and Y. Shin, "Testing the null hypothesis of stationarity against the alternative of a unit root: How sure are we that economic time series have a unit root?," Journal of econometrics, vol. 54, no. 1-3, pp. 159-178, 1992.

[37] G. E. Box, G. M. Jenkins, G. C. Reinsel, and G. M. Ljung, Time series analysis: forecasting and control. John Wiley \& Sons, 2015.

[38] S. Hochreiter and J. Schmidhuber, "Long short-term memory," Neural computation, vol. 9, no. 8, pp. 1735-1780, 1997.

[39] D. P. Kingma and J. Ba, "Adam: A method for stochastic optimization," arXiv preprint arXiv:1412.6980, 2014

[40] F. Minucci, E. Vinogradov, and S. Pollin, "Avoiding collisions at any (low) cost: Ads-b like position broadcast for uavs," Ieee Access, vol. 8, pp. 121843-121857, 2020

[41] S. Zhu, X. Zheng, L. Liu, and H. Ma, "Airsync: Time synchronization for large-scale iot networks using aircraft signals," in 2020 17th Annual IEEE International Conference on Sensing, Communication, and Networking (SECON), pp. 1-9, IEEE, 2020. 